PINTO, L.H.; SOUZA, H.; CARNEIRO, T.K.

REF-ISSN1808-0804 Vol.XII (2),16-29, 2015.

\title{
AVALIAÇÃo DA FREQUÊNCIA DE INTERAÇÕES MEDICAMENTOSAS OCORRIDAS COM PACIENTES INTERNADOS EM CLÍNICA CIRÚRGICA EM UM HOSPITAL PÚBLICO DE JOINVILLE.
}

EVALUATION OF THE FREQUENCY OF DRUG INTERACTIONS OCCURRING IN PATIENTS ADMITTED TO THE SURGICAL CLINIC IN A PUBLIC HOSPITAL OF JOINVILLE.

EVALUACIÓN DE LA FRECUENCIA DE LAS INTERACCIONES MEDICAMENTOSAS OCURRIÓ EN PACIENTES HOSPITALIZADOS EN CLÍNICA QUIRÚRGICA EN UN HOSPITAL PÚBLICO DE JOINVILLE.

\section{Luciano Henrique Pinto ${ }^{1 *}$, Helena de Souza ${ }^{1}$, Tamara Kassandra Carneiro ${ }^{1}$}

Universidade da Região de Joinville - UNIIVILLE

E-mail*: lucianohp.pq@gmail.com

Submetido em: 12/12/2014; Aceito em: 19/05/2015; Publicado em: 30/06/2015

\section{RESUMO}

Interações medicamentosas são definidas como alteração no efeito esperado de certos medicamentos se administrados junto com outros fármacos. Implica em prejuízo nas respostas terapêuticas esperadas, devido a alterações tanto no ponto de vista farmacocinético quanto farmacodinâmico, o que pode vir a ocasionar em prejuízos clínicos no tratamento estabelecido. O presente estudo teve como objetivo verificar a ocorrência de interações medicamentosas clinicamente relevantes no setor de clínica cirúrgica do Hospital Regional Hans Dieter Schmidt. Foram analisados neste estudo o 
PINTO, L.H.; SOUZA, H.; CARNEIRO, T.K.

REF-ISSN1808-0804 Vol.XII (2),16-29, 2015.

prontuário contendo prescrições de 50 pacientes internados na clinica cirúrgica. As interações medicamentosas clinicamente relevantes foram classificadas em graves e moderadas tendo como fonte de dados o site Drugs.com ${ }^{\circledR}$. Foram investigadas 434 prescrições, e identificadas 376 interações, sendo estas 312 moderadas e 64 graves. Dentre as interações classificadas como graves, a que ocorreu com maior frequência foi entre Tramadol e Metoclopramida com 54\% do total de interações graves. A ocorrência de interações medicamentosas é freqüente nos hospitais brasileiros e necessita de maior atenção devido aos riscos em que o paciente é exposto. A criação e implantação de mecanismos que visam diminuir estas ocorrências se fazem necessário e trazem benefícios não somente ao paciente e ao seu respectivo tratamento, mas também aos hospitais, resultando em diminuição de gastos excessivos e desnecessários.

PALAVRAS ChAVE: Medicamentos, Farmacêutico, Hospitalar.

\section{ABSTRACT}

Interactions are defined as changes in the expected effect of certain medications if taken along with other drugs. Implies loss in therapeutic responses expected, due to changes in both the pharmacokinetic viewpoint as pharmacodynamic, which may ultimately result in losses in the clinical treatment established. The present study aimed to verify the occurrence of clinically relevant drug interactions in real surgical clinic of the Hospital Regional Hans Dieter Schmidt. This study evaluated the medical records containing prescriptions for 50 patients admitted to the surgical clinic. Clinically relevant drug interactions were classified as having severe and moderate data source website Drugs.com $® .434$ prescriptions were investigated and identified 376 interactions, which were 312 moderate and 64 severe. Among the interactions were classified as serious, the more often occurred between tramadol and metoclopramide was 54\% of the total serious interactions. The occurrence of drug interactions is common in Brazilian hospitals and needs more attention due to the risk that the patient is exposed. The creation and implementation of mechanisms to reduce these occurrences are warranted and bring benefits not only to the patient and their processing, but also to hospitals, resulting in decrease of overspending and unnecessary.

KEYWORDS: Medications, Pharmaceutical, Hospital. 


\title{
Revista Eletrônica de Farmácia
}

\author{
PINTO, L.H.; SOUZA, H.; CARNEIRO, T.K. \\ REF-ISSN1808-0804 Vol.XII (2),16-29, 2015.
}

\section{RESUMEN}

Las interacciones se definen como cambios en la estimación de los efectos de ciertos medicamentos si se toma junto con otros medicamentos. Implica la pérdida en las respuestas terapéuticas esperados, debido a los cambios, tanto en el punto de vista farmacocinético como farmacodinámico, que pueden en última instancia resultar en pérdidas en el tratamiento clínico establecido. El presente estudio tuvo como objetivo verificar la ocurrencia de interacciones farmacológicas clínicamente relevantes en clínica quirúrgica real del Hospital Regional Hans Dieter Schmidt. Este estudio evaluó los registros médicos que contienen recetas de 50 pacientes ingresados en la clínica quirúrgica. Las interacciones medicamentosas clínicamente relevantes fueron clasificados como severos y moderados de datos de origen página web Drugs.com $® .434$ recetas se investigaron e identificaron 376 interacciones, que eran 312 moderada y 64 severa. Entre las interacciones se clasificaron como graves producido la mayor frecuencia entre tramadol y metoclopramida fue $54 \%$ del total de interacciones graves. La aparición de interacciones de drogas es común en los hospitales brasileños y necesita más atención debido al riesgo de que el paciente está expuesto. La creación e implementación de mecanismos para reducir estos sucesos están garantizados y traerá beneficios no sólo para el paciente y su tratamiento, sino también a los hospitales, lo que resulta en una disminución de gastos excesivos e innecesarios.

PALABRAS ClAVE: Medicamentos, Farmacéutico, Hospital.

\section{INTRODUÇÃO}

Interações medicamentosas são definidas como a alteração no efeito de certo medicamento se administrado junto com outro fármaco ou alimento que implique em prejuízo nas respostas terapêuticas esperadas. As interações podem ser benéficas do ponto de vista farmacocinético e farmacodinâmico ou podem em alguns casos prejudicar o tratamento estabelecido, seja por aumentar o efeito de um fármaco, seja pela redução do efeito deste. Problemas envolvendo interações medicamentosas clínicas relevantes estão ganhando espaço nas discussões clinicas e farmacêuticas, devido a crescente demanda de situações em que se faz necessário um maior conhecimento sobre as interações, além da necessidade de criação de sistemas de verificação de interações adequados. ${ }^{(1)}$ 


\section{Revista Eletrônica de Farmácia}

PINTO, L.H.; SOUZA, H.; CARNEIRO, T.K.

REF-ISSN1808-0804 Vol.XII (2),16-29, 2015.

A atenção aos cuidados farmacológicos, em especial na questão das interações medicamentosas clinicamente relevantes em pacientes internados, vem assumindo uma preocupação diferenciada pelos profissionais de saúde, uma vez que a busca da segurança dos medicamentos - desde a sua escolha até a administração - não pode ser descartada ou subestimada. (2) O fenônemo das interações medicamentosas ocorre com bastante frequencia na prática clínica. Estudos apontam que esta situação varia de 3 a $5 \%$ entre pacientes que apresentaram polifarmácia, podendo chegar a $20 \%$ entre os pacientes que fazem 0 uso diário de mais de 10 drogas, foram citadas como causa de cerca de $3 \%$ das hospitalizações e podem causar vários efeitos adversos aos pacientes. ${ }^{(3)}$

As interações medicamentosas são classificadas como: Menores, Moderadas e Maiores ou Graves. São consideradas menores aquelas que podem causar efeitos clínicos restritos, e em geral não requerem mudanças na terapia medicamentosa. As Moderadas, são aquelas interações que resultam em alterações do quadro clínico do paciente, requerendo mudanças na terapia medicamentosa. As Maiores ou Graves são o tipo de interação que requer intervenção médica para minimizar ou prevenir as reações adversas clinicamente relevantes e de potencial risco de morbidade relacionada ao medicamento (MRM). ${ }^{(2)}$

Pacientes submetidos a procedimentos cirúrgicos, idosos, imunodeprimidos, e internados em unidade de terapia intensiva (UTI), são citados como os grupos mais suscetíveis ao risco de interações medicamentosas, principalmente nas internações hospitalares, onde a complexa condição clinica destes pacientes muitas vezes requer grandes números de medicações a serem administradas. ${ }^{(4)}$

Principalmente em hospitais, é freqüente a relação entre a grande demanda de fármacos prescritos e o risco potencial de interações que ocorrem entre estes medicamentos, o que vem a ser uma problemática presente ainda nos dias de hoje em hospitais públicos e privados. ${ }^{(5)}$

Este estudo tem por objetivo, verificar e discutir a ocorrência de interações medicamentosas clinicamente relevantes na clínica cirúrgica de um Hospital público situado na cidade de Joinville-SC, demonstrando a importância da monitorização dessas ocorrências, e indicando possíveis meios que possam 


\title{
Revista Eletrônica de Farmácia
}

\author{
PINTO, L.H.; SOUZA, H.; CARNEIRO, T.K. \\ REF-ISSN1808-0804 Vol.XII (2),16-29, 2015.
}

ser utilizados para evitar os danos causados por interações medicamentosas graves.

\section{METODOLOGIA}

O presente estudo foi desenvolvido pelo método "estudo de caso" no qual foi feita a descrição e análise da prescrição eletrônica dos pacientes internados na clínica cirúrgica, unidade localizada no interior do Hospital Regional Hans Dieter Schmidt no período de março de 2012. O setor possui 44 leitos ativos e realiza cirurgias gerais, excluindo-se as cardíacas, bariátricas e plásticas, que são realizadas em outros setores do Hospital.

O Hospital Regional Hans Dieter Schmidt - Joinville é uma entidade Pública Estadual pertencente à Secretaria de Estado da Saúde de Santa Catarina, fundado em 15 de março de 1984, sem fins lucrativos, prestadora de serviços de média e alta complexidade, que oferece atendimento Médico/Hospitalar Especializado e têm como referência as especialidades de cardiologia/cirurgias cardíacas, pneumologia/infectologia, cirurgia bariátrica, psiquiatria a toda comunidade de Joinville e região (macro região norte de 800 mil habitantes). O atendimento é $100 \%$ SUS com capacidade para 237 leitos ativos, realizando em média cerca de 8.959 atendimentos por mês, e com uma média de 750 internações por mês. Com a certificação de Hospital Ensino, que ocorreu no ano de 2007, o Hospital passou a ser um centro formador em atenção e pesquisa na área da saúde, focado para as exigências do SUS e dedicado à formação multiprofissional.

A dinâmica deste estudo consistiu em uma análise retrospectiva e transversal com intuído de se obter informações a respeito do:

- Número total de prescrições realizadas no mês,

- Número total de interações medicamentosas totais, independente da classificação, ocorridas no mês,

- Número de interações tidas como "maiores ou graves" ocorridas no mês,

- Interações "maiores ou graves" mais frequentes,

- Número de interações tidas como "moderadas" no mês,

- Tempo médio de internação do paciente, 
PINTO, L.H.; SOUZA, H.; CARNEIRO, T.K.

REF-ISSN1808-0804 Vol.XII (2),16-29, 2015.

- Número médio de pacientes durante o período de estudo,

Foram considerados aptos a participar da pesquisa os pacientes submetidos a cirurgias e posteriormente internados no setor da Clinica Cirúrgica, no período correspondente a março de 2012, que permaneceram por mais de um dia, e utilizavam mais de um medicamento prescrito desde o inicio da internação no setor.

O estudo obedeceu aos seguintes critérios de exclusão:

\section{Crianças}

- Adolescentes menores de 18 anos,

- Pacientes com apenas uma medicação prescrita desde o inicio da internação no setor.

- Pacientes internados por um período menor ou igual á 24 horas.

Dentre todos os pacientes internados na unidade em estudo no período estipulado, obteve-se um total de 50 pacientes aptos a participar do estudo via análise de sua prescrição eletrônica.

O levantamento das interações
medicamentosas contidas nas prescrições, bem como sua classificação de risco foi conduzido tendo como referência as informações referente ao riscos à saúde contidas no site www.drugs.com ${ }^{\circledR}$

As análises estatísticas foram realizadas pelo programa EPIINFO, versão 2000 visando significância estatística das associações com nível de $95 \%$ de confiança.

Este estudo foi submetido ao Comitê de Ética em Pesquisa do Hospital Regional Hans Dieter Schmidt, sob o parecer 002/2013, e aprovado.

\section{RESULTADOS}

O estudo realizado pela análise de prontuário contendo prescrições de 50 pacientes submetidos a cirurgias, apontou que o tempo médio de internação destes pacientes era de aproximadamente \pm 8 dias no setor da clinica cirúrgica do Hospital Regional Hans Dieter Schmidt, no mês de março de 2012. Um período considerado normal dentro das médias encontradas em outros estudos.

O número de prescrições analisadas no período foi de 434 , no qual se teve uma média de $\pm 8,68$ prescrições por paciente. A quantidade de medicamentos prescritos foi de 2.651 substâncias ativas, sendo em média 


$$
\begin{gathered}
\text { PINTO, L.H.; SOUZA, H.; CARNEIRO, T.K. } \\
\text { REF-ISSN1808-0804 Vol.XII (2),16-29, } 2015 .
\end{gathered}
$$

$\pm 53,02$ substâncias ativas prescritas por paciente durante 0 período de internação. Interações classificadas como Graves e Moderadas totalizaram um número de 376 interações, dentre as quais, 64 eram consideradas interações graves - aproximadamente $1 \%$ - e 312 interações moderadas - que corresponde a quase $10 \%$ do total (GRÁFICO 01). A ocorrência das interações moderadas nos valores encontrados apontam para a necessidade de uma atenção especial para a ocorrência deste fenômeno.

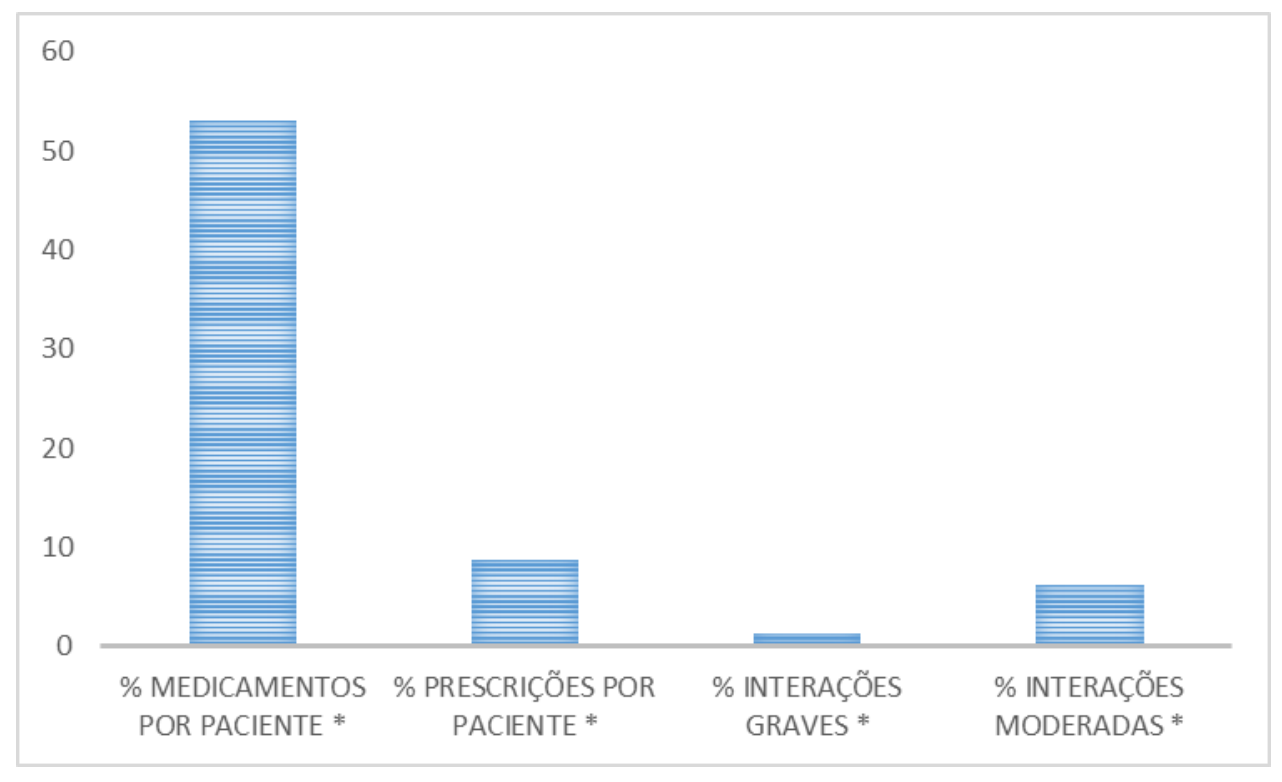

Gráfico 01: Percentual de medicamentos prescritos por paciente, de prescrições realizadas por paciente, do total de interações graves e total de interações moderadas, em função do número de pacientes. * Referente ao período de estudo

Fazendo a análise tendo por parâmetro o total de prescrições realizadas, a incidência de interações medicamentosas classificadas como moderadas corresponderam a $71,8 \%$ em relação ao total de prescrições, seguidas das graves com 14.7\% (GRÁFICO 02). As que não apresentaram interações significativas, ou não apresentaram nenhuma interação aparecem com apenas $13,3 \%$ do total de prescrições. Observa-se com estes resultados que no universo das prescrições realizadas, cerca de $85 \%$ das prescrições apresentavam algum tipo de interação grave ou moderada. Esta alta ocorrência chama a atenção para este fenômeno relativo à interação por prescrição, no qual pode se ter explicação para a questão da necessidade clinica, falta de opções de 
PINTO, L.H.; SOUZA, H.; CARNEIRO, T.K.

REF-ISSN1808-0804 Vol.XII (2),16-29, 2015.

medicamentos ou desconhecimento por das interações.

parte do prescritor a respeito dos riscos

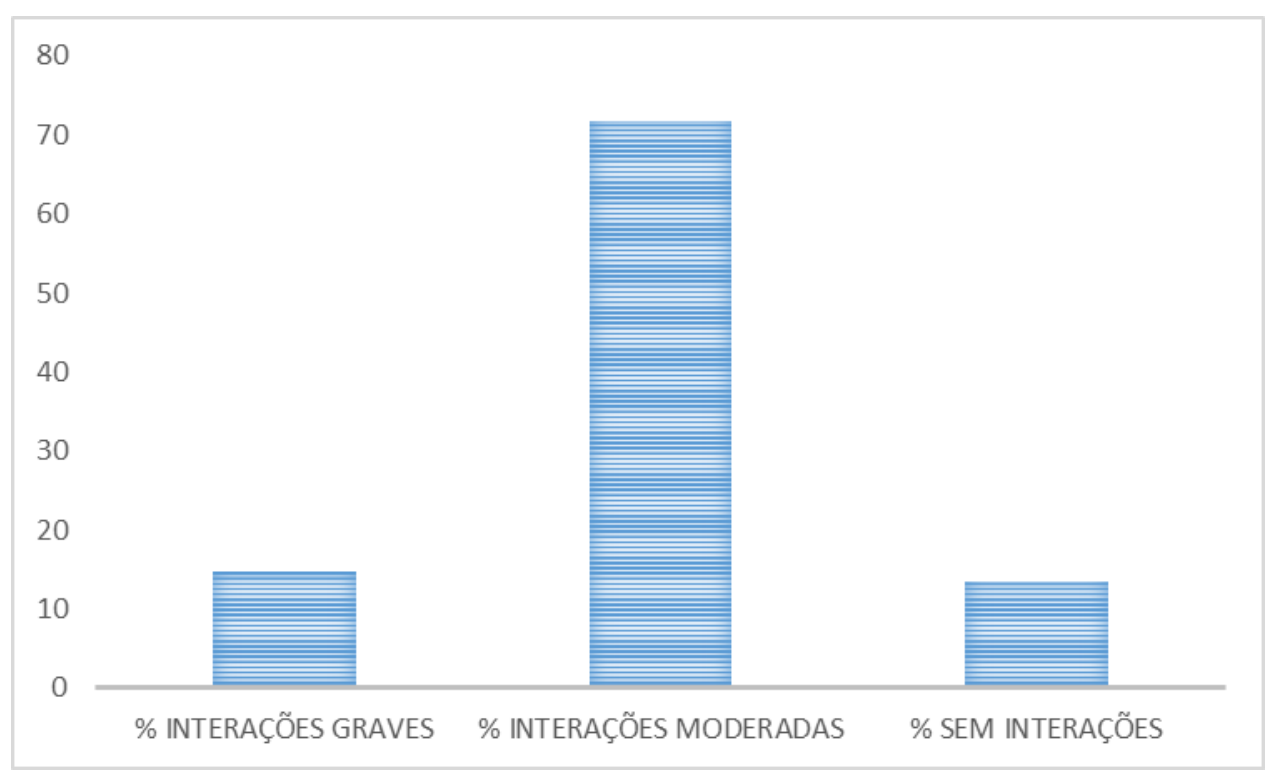

Gráfico 02: Percentual de ocorrência de interações em relação ao total de prescrições realizadas.

Considerando 0 total de prescrições, nota-se que a interação entre metoclopramida e tramadol é a mais freqüente dentre as classificadas como graves (GRÁFICO 03). O uso combinado de tramadol com metoclopramida pode levar a uma potencialização dos efeitos sedativos atribuídos ao tramadol, bem como a ocorrência de outros efeitos indesejados, como o aumento de riscos de convulsões. Tais condições credenciam o uso combinado destes fármacos serem feitos com criteriosa avaliação, pois apenas nos casos em que se tem um potencial beneficio frente a um risco moderado é que se deve fazer tal associação. Outro fator importante e que deve ser destacado, é a questão da condição clinica do paciente, pois uma vez tendo histórico de convulsões é comum a chance de ocorrência de riscos associados a esta interação. 
PINTO, L.H.; SOUZA, H.; CARNEIRO, T.K.

REF-ISSN1808-0804 Vol.XII (2),16-29, 2015.

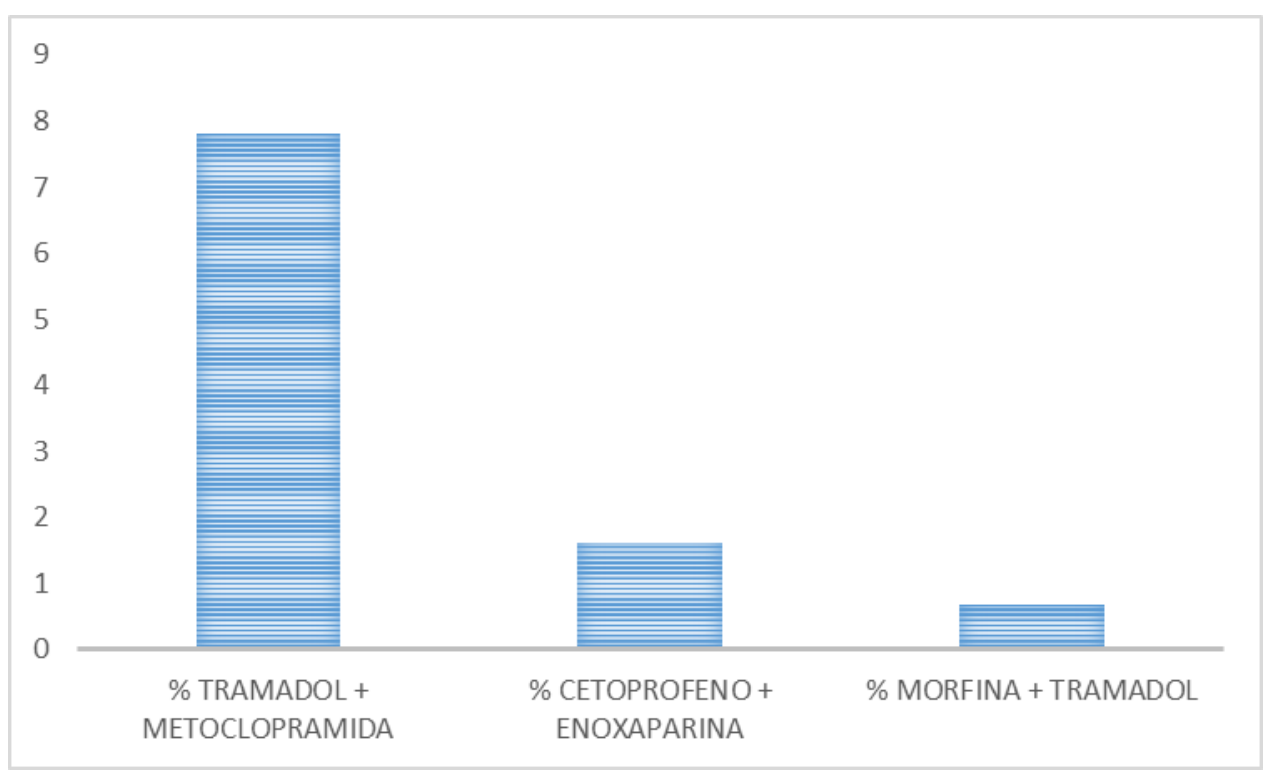

Gráfico 03: Interações graves mais freqüentes em relação ao total de prescrições.

$\mathrm{Na}$ análise feita entre as interações e sua frequência de ocorrência, nota-se que a interação entre tramadol e metoclopramida se repetiu por 34 vezes, representando cerca de $53 \%$ das interações graves que se repetiram durante o estudo, evidenciando a grande ocorrência deste tipo de interação.

\begin{tabular}{ccc}
\hline INTERAÇõES MAIS FREQUENTES & $\begin{array}{c}\mathbf{N}^{\circ} \text { DE } \\
\text { REPETIÇõ ES }\end{array}$ & PERCENTUAL \\
\hline Tramadol + metoclopramida & 34 & $53,10 \%$ \\
Cetoprofeno+enoxaparina & 7 & $10,90 \%$ \\
Morfina + tramadol & 3 & $4,60 \%$
\end{tabular}

Tabela 04: Número de repetições das interações graves mais freqüentes, apresentadas em valores bruto e relativo.

\section{DISCUSSÃo}

O estudo chama a atenção para o número de ocorrências de interações medicamentosas nas prescrições feitas aos pacientes submetidos a algum tipo de procedimento cirúrgico e posteriormente internados. De acordo com os dados apresentados no Gráfico 01, o número de interações significativas ocorridas chega quase a se igualar ao total de prescrições/dia por paciente, sendo assim, em media todos os pacientes submetidos ao estudo foram expostos a pelo menos 1 interação grave e 6 interações moderadas no período de 
PINTO, L.H.; SOUZA, H.; CARNEIRO, T.K.

REF-ISSN1808-0804 Vol.XII (2),16-29, 2015.

8 dias de internação. Em cerca de 85\% das prescrições analisadas, houve ao menos 1 interação medicamentosa potencial, esse número ultrapassa ao descrito por Sehn et al. ${ }^{(6)}$, que relatou um valor de $65 \%$ em estudo semelhante realizado em outro hospital público brasileiro no ano de 2003. Os dados encontrados, principalmente no que se refere a quase proporção de 1 interação moderada por dia chama a atenção para o risco em que pode estar exposto o paciente, e no qual tal condição acaba sendo subestimada.

A frequente ocorrência da interação entre tramadol e metoclopramida, com $54 \%$ das ocorrências em relação ao total de interações graves, levanta a questão sobre a origem deste tipo de interação: se ela se baseia na necessidade pós avaliação risco beneficio ou pelo simples ato prático de escolha sem uma avaliação criteriosa. Mesmo que esta associação seja bastante comum devido a grande incidência de náuseas e vômitos descritas como efeito adverso ao tramadol, algumas literaturas apontam que esta interação é potencialmente perigosa e sugerem maior atenção no monitoramento do paciente hospitalizado, principalmente em pacientes com função renal comprometida, função hepática comprometida ou com históricos de convulsão. ${ }^{(7,19)}$

Pacientes expostos a interações medicamentosas - sejam moderadas ou graves - estão em uma condição denominada morbidade relacionada ao medicamento (MRM), pois a combinação dos fármacos pode levar ao fracasso de um dos agentes terapêuticos, ou levar a produção de um novo problema clinico relacionado a exacerbação do efeito de um dos medicamentos em uso. ${ }^{(8)}$

Pequena parte destas MRMs é imprevisível, enquanto que a grande maioria seria totalmente previsível e passível de prevenção, como as que são originadas por interações medicamentosas. ${ }^{(8)}$

Questões relativas à MRM e ocorrência de interações medicamentosas não são recentes. Em 1976, McKenney \& Harrison $^{(9)}$ relataram que 59 de 216 admissões em uma unidade médica cirúrgica incluíam situações de MRMs. Em 1977 Porter \& Jick ${ }^{(10)}$ concluíram que a taxa de mortalidade devida a MRMs nos Estados Unidos era de 1,2 mortes para cada 1000 admissões hospitalares. Cerca de $1 \%$ levaram a mortes relacionadas com medicamentos, dos quais $25 \%$ eram evitáveis - sendo 


\title{
Revista Eletrônica de Farmácia
}

\author{
PINTO, L.H.; SOUZA, H.; CARNEIRO, T.K. \\ REF-ISSN1808-0804 Vol.XII (2),16-29, 2015.
}

algumas delas originadas a partir de interações medicamentosas.

Quanto ao Brasil, é evidente a carência de trabalhos de investigação sobre a morbidade e mortalidade associada ao uso de medicamentos, o que inclui a interação medicamentosa. Fatores que contribuem para isto é a subutilização do sistema da Farmacovigilância no país - quando comparado com outros países - e a falta de hábito dos profissionais de saúde em registrar notificações de queixa sobre os medicamentos. Fatos estes que comprometem a aquisição de um diagnóstico preciso do problema no Brasil. ${ }^{(11)}$

Em se tratando de situações onde existe pouca avaliação ou critérios de escolha dos medicamentos em uso, é necessário disciplinar o corpo de prescritores, tornando como principio o uso dos Protocolos farmacoterapeuticos, que se fundamenta na lógica do Uso Racional de Medicamentos (URM). Dentro da lógica do URM, um ensaio clinico randomizado, duplo cego, controlado por placebo supera de longe a opinião de um único especialista e sua experiência clinica. A força de evidência para a ação de um medicamento, risco ou risco decorrente de interação medicamentosa depende da qualidade e da origem de sua fonte de informação utilizada, e que vai gerar um protocolo de diretrizes terapêuticas ${ }^{(12)}$.

A criação de protocolos e rotinas para a prescrição é uma grande alternativa para auxiliar na diminuição de fatores que levam a falhas ao tratamento medicamentoso do paciente. ${ }^{(13)}$ Além de protocolos, alguns autores recomendam a utilização de programas informatizados para a avaliação da prescrição médica com a finalidade de detectar possíveis interações a serem prescritas, mas também frisam a importância do farmacêutico clínico em avaliar e controlar os medicamentos prescritos dentro dos hospitais, pois existe ainda a possibilidade de interação com medicamentos de uso continuo já utilizados pelos pacientes diariamente, e que possivelmente não são identificados por estes programas. ${ }^{(6)}$ A prática da Farmácia Clinica que teria por finalidade aumentar a efetividade e segurança do tratamento medicamentoso ${ }^{(14)}$, poderia ser útil tanto em nível de análise de prescrição, como na colaboração da elaboração de protocolos, que visariam prevenir e impedir ocorrência de possíveis interações medicamentosas dentro dos hospitais e que muitas vezes levam a graves consequências. ${ }^{(15)}$ 
PINTO, L.H.; SOUZA, H.; CARNEIRO, T.K.

REF-ISSN1808-0804 Vol.XII (2),16-29, 2015.

O farmacêutico detém conhecimentos importantes em farmacologia e toxicologia, desta forma em âmbito hospitalar é o profissional de saúde mais indicado para realizar a identificação e monitoramento de possíveis interações medicamentosas em prescrições, sendo este profissional indispensável para a diminuição dos riscos associados a terapêutica prescrita.(16) Em um estudo realizado por Reis et al. ${ }^{(17)}$ no Hospital de Clínicas da Universidade Federal do Paraná, foi constatado que as intervenções realizadas pelo Farmacêutico clinico, promoveram mudanças benéficas em 7 de cada 10 prescrições que apresentavam algum tipo de PRM, dentre os quais, $7,5 \%$ do total correspondiam à interações medicamentosas.

No levantamento de dados não
foram realizadas análises dos prontuários em nível investigatório, a fim de evidenciar possíveis reações causadas pelas interações medicamentosas, entretanto de acordo com estudos previamente publicados, as interações entre dois ou mais medicamentos em uma mesma prescrição, devem ser investigadas e monitoradas mesmo não havendo uma manifestação clínica imediata, pois a suspeita inicial serve para deixar a equipe médica em alerta, e agir de forma imediata frente à ocorrência de reações indesejadas. ${ }^{(18)}$

\section{CONCLUSÃO}

Com os resultados obtidos neste estudo foi possível demonstrar a grande ocorrência de interações existentes entre as medicações prescritas, e chamar a atenção sobre a necessidade de padronizar medidas preventivas dentro dos hospitais. As interações medicamentosas relevantes causam deficiência na terapêutica podendo prejudicar a mesma. A falta de verificação destas interações pode ainda gerar custos extras para o hospital para tratamento de complicação devido à interação, ou mesmo novo medicamento para auxiliar a terapia. Alem de preocupação com equipamentos e profissional treinado, os hospitais devem ter atenção redobrada com os medicamentos utilizados na terapia, sua segurança e possíveis efeitos que a mesma possa trazer ao paciente. É necessário ainda que se realizem

mais estudos indicativos e com evidencia s clinicas para melhor auxiliar na analise deste problema, buscando despertar mai or interesse na criação e implantação de mecanismos que possam identificar adequar 


\title{
Revista Eletrônica de Farmácia
}

\author{
PINTO, L.H.; SOUZA, H.; CARNEIRO, T.K. \\ REF-ISSN1808-0804 Vol.XII (2),16-29, 2015.
}

$\begin{array}{llrl}\text { conseqüentemente } & \text { vir } & \text { a } & \text { hospitais, e ressaltar a importância do } \\ \text { resultar na diminuição } & & \text { destas } & \text { profissional }\end{array}$

freqüências no ambiente hospitalar. Este

farmacêutico como colaborador

estudo espera ainda contribuir com responsável por promover uso racional

dados relevantes para futuras de medicamentos, e auxiliar na discussões a respeito da ocorrência de elaboração e implantação de protocolos interações medicamentosas dentro dos clínicos junto à equipe multidisciplinar.

\section{REFERÊNCIAS}

1. Toffoletto MC, Padilha KG. Conseqüências de Medicação em Unidades de Terapia Intensiva e Semi-Intensiva. Rev. Esc. Enferm. USP. 2006; 40(2): 247-52.

2. Yunes LP, Coelho TA, Almeida SM. Principais Interações Medicamentosas em Pacientes da UTI Adulto de um Hospital Privado de Minas Gerais. Rev. Bras. Farm. Hosp. Saúde São Paulo, 2011; v. 2 n. $323-26$.

3. Bleich GW, Bleich A, Chiamulera P, Sanches ACC, Schneider DSLG, Teixeira JJV. Frequência de Potenciais Interações entre Drogas nas Prescrições Médicas em uma Cidade do Sul do Brasil. Sao Paulo Med. J. 2009; 127( 4 ): 206-210.

4. Lima REF. Interações Medicamentosas Potenciais em Pacientes de Unidade de Terapia Intensiva de um Hospital Universitário do Ceará. [dissertation]. Ribeirão Preto-SP: Universidade de São Paulo/USP; 2007. 116p.

5. Kawano DF, Pereira LRL, Ueta JM, Freitas O. Acidentes com Medicamentos: como minimizá-los? Rev. Bras. Ciên. Farm. 2006; vol. 42, n. 4.

6. Sehn R, Camargo AL, Ferreira MBC. Interações Medicamentosas Potenciais em Prescrições de Pacientes Hospitalizados. Infarma. 2003; vol.15 $\mathrm{n}^{\circ} 9-10$. Available from: http://www.cff.org.br/sistemas /geral/revista/pdf/86/infarma007.pdf

7. Santos TOD, Estrela TG, Azevedo VLF, Oliveira OEC, Oliveira Júnior G, Figueiredo GS. Uso do Tramadol Venoso e Subcutâneo em Herniorrafia Inguinal: Estudo Comparativo. Rev. Bras. Anestesiol. 2010; 527 Vol. 60.

8. Cipolle RJ, Strand LM, Morley PC. O exercício do cuidado farmacêutico. Conselho Federal de Farmácia [Internet]. 2006 [cited 2009 ago 26]; 378 p.

9. Mckelley JM, Harrison WL. Drug-Related Hospital Admissions. American Journal of HealthSystem Pharmacy, 1976; vol. 33 no. 8 792-795.

10. Porter J, Jick H. Drug-Related Deaths. The Journal of the American Medicinal Association. JAMA. 1977; Vol. 237 No. 23.

11. Sistema Nacional de Informações Tóxico Farmacológicas [internet]. Rio de Janeiro: Sinitox divulga novos dados de intoxicação humana [cited 2009]. Available from:http://www.fiocruz.br/sinitox_novo/cgi/cgilua.exe/sys/start.htm?infoid=105\&sid=107

12. Fuchs, F.D.; Wannmacher, L. Farmacologia Clínica Fundamentos da Terapêutica Racional . Editora Guanabara Koogan, 3a edição, 2010 


$$
\begin{gathered}
\text { PINTO, L.H.; SOUZA, H.; CARNEIRO, T.K. } \\
\text { REF-ISSN1808-0804 Vol.XII (2),16-29, } 2015 .
\end{gathered}
$$

13. Souza JMC, Thomson JC, Catisti DG. Avaliação de prescrições medicamentosas de um hospital universitário brasileiro. Rev. Bras. Educ. Med. 2008; 32 (2) : 188 - 196

14. Oliveira AB, Oyakawa CN, Miguel MD, Zanin SMW; Montrucchio DP. Obstáculos da Atenção Farmacêutica no Brasil. Rev. Bras. Cienc. Farm. 2005; 41( 4 ): 409-413.

15. Santos HC, Ribeiro RR, Ferrarini M, Fernandes JPS. Possíveis Interações Medicamentosas com Psicotrópicos Encontrados em Pacientes da Zona Leste de São Paulo. Rev. Ciênc. Farm. Básica Apli. 2009; 30(3):285-289 ISSN 1808-4532.

16. Sobrinho FF, Nascimento JWL. Avaliação de Interações Medicamentosas em Prescrições de Pacientes Hospitalizados. Revista Racine Ed.94. 2006.

17. Reis WCT, Scopel CT, Correr CJ, Andrzejevski VMS. Análise das intervenções de farmacêuticos clínicos em um hospital de ensino terciário do Brasil. Einstein (São Paulo). v. 11, n. 2.

18. Silva NMO, Carvalho RP, Bernardes ACA, Moriel P, Mazzola PG, Franchini CC. Avaliação de potenciais interações medicamentosas em prescrições de pacientes internadas, em hospital público universitário especializado em saúde da mulher, em Campinas-SP. Rev. Ciênc. Farm. Básica Apl. $2010 ; 31(2): 171-176$ ISSN 1808-4532.

19. Riechelmann R.P., Tannock I.F., Wang L., Saad E.D., Taback N.A., Krzyzanowsk M.K. Potential drug interactions and duplicate prescriptions among cancer patients. J. Natl. Cancer Inst. 2007;99:592-600. doi: 10.1093/jnci/djk130 